\title{
Can Single Positive Core Prostate Cancer at biopsy be Considered a Low-Risk Disease after Radical Prostatectomy?
}

Ricardo Kupka da Silva, Marcos Francisco Dall’Oglio, Alexandre Crippa Sant’Ana, José Pontes Júnior, Miguel Srougi

Division of Uro-Oncology, University of São Paulo Medical School, São Paulo, SP, Brazil

\section{ABSTRACT}

Purpose: Single positive core in a prostate biopsy is usually associated with indolent prostate cancer (PCa) and is one of the active surveillance (AS) inclusion criteria. We investigated whether single positive core PCa at biopsy could define an archetype of low-risk disease.

Materials and Methods: A total of 1320 consecutive patients were enrolled. Among them, 249 patients with single positive core PCa were followed up, and the clinical and pathological parameters influencing prognosis were analyzed.

Results: Out of the 249 patients, 172 (69.0\%) had pathological findings $\geq$ pT2c and 87 (34.9\%) had an undergraded Gleason Score (GS) based on the biopsy. Positive surgical margins (PSMs), extraprostatic extension (EPE) and seminal vesicle invasion (SVI) were found in $20.8 \%, 10.0 \%$ and $6.0 \%$ of patients, respectively. In a comparative analysis, we found that the PSA level, prostate weight and number of cores at biopsy are essential to correctly predict an indolent PCa. A total of 125 patients (67.3\%) with nonpalpable tumors became high-risk tumors (pT2c-T3). Analyzing only nonpalpable tumors with a GS of 6 at biopsy (156 patients), we noted that 106 (67.9\% of cT1) progressed from cT1c to pT2c-pT3.

Conclusions: Single core PCa have clinically significant disease in the Radical Prostatectomy specimens, with considerable rates of overgrading for the GS, pT2c-pT3, PSMs, EPE and SVI. The treatment plan must be evaluated individually for patients with single core PCa and must take into account other prognostic factors when determining whether a patient should be managed with AS.

\section{ARTICLE INFO}

\section{Key words:}

Prostatic Neoplasms; Neoplasm Staging; Prostate-Specific Antigen; Neoplasm Grading; Biopsy, Needle

Int Braz J Urol. 2013; 39: 800-7

Submitted for publication: April 09, 2013

Accepted after revision: September 04, 2013

\section{INTRODUCTION}

Prostate cancer $(\mathrm{PCa})$ remains the most common noncutaneous cancer and the second leading cause of cancer death among men in Western countries (1). Its prevalence in men older than 50 years has been estimated to be as high as $40 \%$ in the USA (2), with an estimated 241,740 new cases and 28,170 deaths related to PCa in 2012
(1). Although the helpfulness of prostate-specific antigen (PSA) screening is still debated (3), its widespread use over the last several decades resulted in increases in PCa diagnoses and stage migration (4). Currently, approximately 75\% of patients have nonpalpable tumors, and only 5\% have metastases at the time of diagnosis. It is not uncommon for tumors to be diagnosed by a single positive core in the prostate biopsy. It is expected that many of 
these single positive core patients may have clinically insignificant PCa characterized by a low volume, early stage and low Gleason score (GS), making them candidates for active surveillance (AS) or watchful waiting (5-7).

Patients with localized disease are treated by radical prostatectomy, radiation therapy or AS (8). The recent publication of two randomized studies of radical prostatectomy versus AS with contradictory results (9-11) increased the controversy regarding the benefits of PCa treatment and indicated that not all patients should be treated, which renewed interest in AS. Delayed treatment for patients with tumor progression is indicated for those with low-volume and well-differentiated tumors (12).

The decision to treat patients who are being monitored by AS is often based on several clinicopathological features such as the PSA level, an increase in the GS, an increase in the tumor volume on repeat biopsy or patient preference (13). Previously published studies have shown that AS does not appear to compromise biochemical or pathological outcomes in appropriately selected patients (14). Despite the scarcity of relevant literature, the finding of a single positive core in the prostate biopsy is one of the factors that qualifies a patient as a candidate for AS. Given the lack of consensus, can we determine if single positive core PCa really represents an indolent tumor?

The purpose of this study was to evaluate the clinical and pathological outcomes of patients with prostate cancer who had only one positive needle biopsy core and underwent radical prostatectomy.

\section{MATERIALS AND METHODS}

The study was submitted to and approved by the ethics committee of HCFMUSP under the number 9201 and was an observational and retrospective analysis. We retrospectively reviewed the cases of all patients who underwent radical retropubic prostatectomies (RRP) for localized PCa at São Paulo's State Cancer Institute (ICESP) between January 2007 and November 2011. We identified 1320 patients, among whom 266 were diagnosed based on a single positive core in the prostate biopsy. Patients who underwent transrectal biopsy with $>10$-core were included in this series (lateral apex, lateral mid-gland, lateral base), as described by Hodge et al (15). In this series, prostate biopsy standardization of the patients diagnosed by repeated biopsy were excluded. Patients who underwent prior hormone treatment, radiotherapy or any other ablative technique before RRP; patients for whom the data were incomplete or missing; and patients with stage pTo cancer were excluded from the study (17 patients). A total of 249 patients were found to meet the aforementioned criteria. The preoperative assessment consisted of medical history, physical examination (including a digital rectum exam), determination of the clinical stage, measurement of the PSA level and review of the prostate biopsy data. The pathological data from the RRP specimens included the GS, the surgical margin status, the presence of extraprostatic extension and the presence of seminal vesicle involvement. Preoperative imaging, such as computed tomography of the pelvis and bone scans, was performed depending on the recommendations of the referring physician.

RRP specimens were submitted for histopathological examination by an experienced group of uropathologists at our institution. The 2002 TNM classification system was used for staging PCa. The 2005 International Society of Urological Pathology (ISUP) guidelines for GS assignment were employed to analyze all pathology specimens. Accordingly to the literature, the adoption of the latest ISUP guidelines improved the agreement of the grading system from 58\% to $72 \%$ (16). According to the new recommendations, there should not be any GS 2-4 cancers in the pathological reports (17). The GS was categorized as 6 or less, 7 or 8 to 10 .

We evaluated the rates of concordance and discrepancy between the preoperative and postoperative GSs. The difference in the GSs was analyzed and divided into undergrading and overgrading categories. Extraprostatic extension (EPE) was defined as neoplastic cells in contact with periprostatic fat. A positive surgical margin (PSM) was defined as the presence of cancer cells in contact with the inked surface.

The patients' characteristics are presented as frequencies (\%) and means \pm SD. Comparisons 
of the clinicopathological features, e.g., clinical stage, PSA level, GS and characteristics of the RRP specimens, were performed using the chi-square and Mann-Whitney tests.

\section{RESULTS}

The baseline characteristics of the study patients are presented in Table- 1 . Some points that are worth noting are that 172 patients $(69.0 \%)$ had a pathological finding $\geq$ pT2c, 87 (34.9\%) had an undergraded GS at biopsy, 20.8\% had PSMs, 10.0\% had EPE and 6.0\% had SVI.

Table-2 shows the GS concordance rates according to the PSA level (categorized as $<10$ or $\geq 10 \mathrm{ng} / \mathrm{mL}$ ) and the prostate weight (categorized as $<50 \mathrm{~g}$ or $\geq 50 \mathrm{~g}$ ). In the group with prostates weighing less than $50 \mathrm{~g}$, patients with a PSA level $<10 \mathrm{ng} / \mathrm{mL}$ had a significantly higher proportion of concordant GSs compared with patients with a PSA level $\geq 10 \mathrm{ng} / \mathrm{mL}(\mathrm{p}=0.024)$. In contrast, the rates of GS concordance and discrepancy did not differ according to the PSA level for patients with prostates weighing more than $50 \mathrm{~g}(\mathrm{p}=0.544)$.

Table- 3 shows that $67.3 \%$ (125) of single positive core $\mathrm{PCa}$ patients with nonpalpable tumors were found to have high-risk tumors (pT2c or pT3) in the pathology analysis. When analyzing only nonpalpable tumors with GS 6 at biopsy (156 patients), we noted that 106 (67.9\% of cT1) went from cT1c to pT2c-pT3 (Table-4).

\section{DISCUSSION}

To our knowledge, this study is the reported largest case series of single positive core PCa patients. We found that these patients may not be ideal candidates for watchful waiting or AS as one-third had undergraded GSs, 20.8\% had PSMs, 10.0\% had EPE and 6.0\% had SVI.

Although the clinical implications of single positive core PCa have not been clearly determined, the incidence of single positive core PCa appears to be increasing in the PSA screening era (5). This "indolent" tumor was initially considered a low-volume PCa. This finding raised concerns that prostatectomies for these patients may be overtreatment. In specific and selected cases, we
Table 1 - Baseline characteristics of 249 patients with single core PCa.

\begin{tabular}{|c|c|}
\hline & Results \\
\hline No. of patients & 249 \\
\hline Mean age (SD) & $65.5(6.4)$ \\
\hline Mean ng/mL PSA (SD) & $9.76(7.1)$ \\
\hline$<10$ & $166(66.7 \%)$ \\
\hline$\geq 10$ & $83(33.3 \%)$ \\
\hline Mean weight g prostate (SD) & $55.7(26.7)$ \\
\hline$<50$ & $127(51 \%)$ \\
\hline$\geq 50$ & $122(49 \%)$ \\
\hline \multicolumn{2}{|l|}{ Clinical Stage } \\
\hline cT1 & $186(74.7)$ \\
\hline cT2 & $60(24.1)$ \\
\hline cT3 & $3(1.2)$ \\
\hline \multicolumn{2}{|l|}{ Biopsy GS } \\
\hline$\leq 6$ & $206(82.7 \%)$ \\
\hline 7 & $30(12.0 \%)$ \\
\hline$\geq 8$ & $13(5.2 \%)$ \\
\hline \multicolumn{2}{|l|}{ RRP Specimens GS } \\
\hline$\leq 6$ & $142(57 \%)$ \\
\hline 7 & $97(39.0 \%)$ \\
\hline$\geq 8$ & $10(5.0 \%)$ \\
\hline \multicolumn{2}{|l|}{ RRP stage } \\
\hline $\mathrm{pT} 2 \mathrm{a}$ & $68(27.3 \%)$ \\
\hline $\mathrm{pT} 2 \mathrm{~b}$ & $9(3.6 \%)$ \\
\hline pT2c & $140(56.2 \%)$ \\
\hline pT3a & $16(6.4 \%)$ \\
\hline pT3b & $16(6.4 \%)$ \\
\hline \multicolumn{2}{|l|}{ Comparison between GS of biopsy and RRP } \\
\hline Concordant & $137(55 \%)$ \\
\hline Undergrading & $87(34.9 \%)$ \\
\hline Overgrading & $25(10.1 \%)$ \\
\hline Positive Surgical margins according to biopsy GS (total) & $52(20.9 \%)$ \\
\hline$\leq 6$ & $40(19.4 \%)$ \\
\hline 7 & $6(20.0 \%)$ \\
\hline$\geq 8$ & $6(46.2 \%)$ \\
\hline Extraprostatic Extension according to biopsy GS (total) & $25(10 \%)$ \\
\hline$\leq 6$ & $19(9.2 \%)$ \\
\hline 7 & $3(10.0 \%)$ \\
\hline$\geq 8$ & $3(23.1 \%)$ \\
\hline $\begin{array}{l}\text { Seminal Vesicle Invasion according to biopsy GS } \\
\text { (total) }\end{array}$ & $15(6.0 \%)$ \\
\hline$\leq 6$ & $12(5.8 \%)$ \\
\hline 7 & $2(6.7 \%)$ \\
\hline$\geq 8$ & $1(7.7 \%)$ \\
\hline
\end{tabular}


Table 2 - Coincidence and types of discrepancy of GS according prostatic weight.

\begin{tabular}{|c|c|c|c|c|c|c|c|c|c|}
\hline \multirow[t]{3}{*}{ Prostate weight } & \multirow[t]{3}{*}{ PSA levels } & \multicolumn{6}{|c|}{ Gleason } & \multicolumn{2}{|c|}{ Total } \\
\hline & & \multicolumn{2}{|c|}{ Undergrading } & \multicolumn{2}{|c|}{ Coincidents } & \multicolumn{2}{|c|}{ Overgrading } & \multirow[b]{2}{*}{$\mathrm{n}$} & \multirow[b]{2}{*}{$\%$} \\
\hline & & $\mathrm{n}$ & $\%$ & $n$ & $\%$ & $\mathrm{~N}$ & $\%$ & & \\
\hline \multirow[t]{3}{*}{$<50 \mathrm{~g}$} & $<10$ & 29 & 31.5 & 57 & 62.0 & 6 & 6.5 & 92 & 100 \\
\hline & $\geq 10$ & 20 & 57.1 & 14 & 40.0 & 1 & 2.9 & 35 & 100 \\
\hline & Total & 49 & 38.6 & 71 & 55.9 & 7 & 5.5 & 127 & 100 \\
\hline \multirow[t]{3}{*}{$\geq 50 \mathrm{~g}$} & $<10$ & 21 & 28.4 & 43 & 58.1 & 10 & 13.5 & 74 & 100 \\
\hline & $\geq 10$ & 17 & 35.4 & 23 & 47.9 & 8 & 16.7 & 48 & 100 \\
\hline & Total & 38 & 31.1 & 66 & 54.1 & 18 & 14.8 & 122 & 100 \\
\hline
\end{tabular}

GS = Gleason score; PSA = Prostate-specific antigen; Undergrading = Biopsy GS less than RRP specimens; Coincidents = Biopsy GS equal to RRP specimens. Overgrading $=$ Biopsy GS greater than RRP specimens

Table 3 - Relationship between clinical stage and pathological stage in single core PCa.

\begin{tabular}{|c|c|c|c|c|c|c|c|c|}
\hline \multirow[t]{3}{*}{ Clinical Stage } & \multicolumn{6}{|c|}{ Pathological Stage } & \multicolumn{2}{|c|}{ Total } \\
\hline & \multicolumn{2}{|c|}{ pT2a-b } & \multicolumn{2}{|c|}{ pT2c } & \multicolumn{2}{|c|}{ pT3 } & \multirow[b]{2}{*}{$n$} & \multirow[b]{2}{*}{$\%$} \\
\hline & $\mathrm{n}$ & $\%$ & $\mathrm{n}$ & $\%$ & $\mathrm{~N}$ & $\%$ & & \\
\hline cT1 & 61 & 32.8 & 105 & 56.5 & 20 & 10.8 & 186 & 100 \\
\hline cT2a-b & 15 & 27.7 & 30 & 55.5 & 9 & 16.7 & 54 & 100 \\
\hline cT2c & 0 & 0.0 & 5 & 83.3 & 1 & 16.7 & 6 & 100 \\
\hline cT3 & 1 & 33.3 & 0 & 0.0 & 2 & 66.7 & 3 & 100 \\
\hline Total & 77 & 30.9 & 140 & 56.2 & 32 & 12.9 & 249 & 100 \\
\hline
\end{tabular}

$\mathbf{P C a}=$ prostate cancer $; \mathbf{G S}=$ Gleason score .

Table 4 - Relationship between clinical stage and pathological stage in single core PCa with GS $\leq \mathbf{6}$.

\begin{tabular}{|c|c|c|c|c|c|c|c|c|}
\hline \multirow[t]{3}{*}{ Clinical Stage } & \multicolumn{6}{|c|}{ Pathological Stage } & \multirow{2}{*}{\multicolumn{2}{|c|}{ Total }} \\
\hline & \multicolumn{2}{|c|}{ pT2a-b } & \multicolumn{2}{|c|}{ pT2c } & \multicolumn{2}{|c|}{ pT3 } & & \\
\hline & $n$ & $\%$ & $\mathrm{n}$ & $\%$ & $\mathrm{~N}$ & $\%$ & $n$ & $\%$ \\
\hline cT1 & 50 & 32.1 & 88 & 56.4 & 18 & 11.5 & 156 & 100 \\
\hline cT2a-b & 11 & 26.2 & 26 & 61.9 & 5 & 11.9 & 42 & 100 \\
\hline cT2c & 0 & 0.0 & 5 & 100.0 & 0 & 0.0 & 5 & 100 \\
\hline cT3 & 1 & 33.3 & 0 & 0 & 2 & 66.7 & 3 & 100 \\
\hline Total & 62 & 30.1 & 119 & 57.8 & 25 & 12.1 & 206 & 100 \\
\hline
\end{tabular}

$\mathbf{P C a}=$ prostate cancer; $\mathbf{G S}=$ Gleason score . 
can consider active surveillance or watchful waiting. The decision to provide treatment for these cases is often based on a combination of variables, with the single positive core in the prostate biopsy being one of these factors (12). However, can single positive core PCa actually be considered a low-risk disease?

The definition of potentially clinically insignificant PCa is controversial, and this category of tumors was originally based on pathological findings of RRP specimens from nonpalpable tumors with favorable pathological characteristics (18). Kerkhof et al. defined good-risk PCa as a GS of 6 or lower, a PSA level of $10 \mathrm{ng} / \mathrm{mL}$ or lower and a stage of cT1-cT2a (19). Most of these patients have low-volume, indolent and slow-growing tumors (5). When we looked in detail at the main features of these single positive core tumors, we noted that the variables used to stratify the risk of aggressiveness already varied preoperatively and after analysis of the RRP specimens.

We categorized the PSA levels, and one-third had a PSA level $>10 \mathrm{ng} / \mathrm{mL}$. This result can be partly explained by the inclusion of men with larger prostates (49\% with prostates $\geq 50 \mathrm{~g}$ ) due to benign prostatic hyperplasia. Using only the PSA criteria, approximately one-third of these single positive core tumors cease to be indolent, and this fact most likely helps explain why we found that $69 \%$ of our patients had a stage $\geq$ pT2c. In his investigation, Brawer concluded that the positive yield of systematic sector biopsies decreases significantly when the prostate volume is greater than 55.6 cc (20); Karakiewicz came to a similar conclusion (21).

We found no significant difference in the prevalence of single positive core PCa according to the prostate weight, most likely because some men with large glands undergo biopsy due to an elevated PSA resulting from benign prostate hyperplasia and not from a significant cancer. This finding could also be explained by the different variables associated with the biopsy, including the gauge of the biopsy needle, the amounts of normal tissue and cancer in the biopsy specimen, the sampling method and interobserver variations (22). The highest concordance between biopsy and Radical Prostatecomy analysis in Single Positive Core Pa- tients occurs when the patient has a low-volume prostate and low PSA level. This is a valuable information since these patients (low-volume prostate, low PSA level and single positive core PCa) must correspond to cases with low-risk disease.

The distribution of clinical stages was very similar to that reported by Sved, who studied 451 patients with low-risk PCa and a GS of 6 at biopsy (23). He reported percentages of 75.4\%, 24.2\% and $0.4 \%$ for cT1, cT2 and cT3, respectively. Unlike these two studies, which evaluated only low-risk populations, the study of Karavitakis et al. found $48 \%$ cT $1,36 \%$ cT2 and 16\% cT3 in a cohort of 95 patients with localized PCa (24).

Regarding the pathological stage, there were 217 patients (87.1\%) with pT2 disease and $32(12.8 \%)$ patients with pT3 disease. Ishizaki et al. studied 144 consecutive patients with localized or locally advanced PCa and observed that the pathological stage tended to be skewed to a lower stage (65.3\% pT2 and 33.9\% pT3 (25). The concordance of GS between the biopsy and RRP specimens in this study (55\%) was similar to that found in two previous studies $(22,26)$, in which the authors found concordance rates of $56 \%$ and $62 \%$, respectively.

Discordance in the GS between biopsy and RRP specimens is widely recognized, but the prognostic importance of these discrepancies has not been definitively established. However, Boorijan et al. (27) published one of the few studies showing that discordance in the GS is associated with adverse pathological features, including advanced tumor stage, lymph node metastasis and positive surgical margins.

Our present study found that $34.9 \%$ of tumors were undergraded and 10.1\% were overgraded, values that are comparable to those published in the literature; Divrik reported exact results of 34\% and 10\%, respectively. Many factors can influence the reliability and interpretation of the agreement in the GS between the biopsy and RRP specimens, such as the biopsy technique, the number of samples and the number and expertise of the pathologists (16). Table-2 shows that GS discordance increased with PSA levels $>10 \mathrm{ng} / \mathrm{mL}$ independent of tumor weight in our single positive core population. 
The GS concordance rate was statistically significant for patients with $<50 \mathrm{~g}$ prostates and PSA levels $<10 \mathrm{ng} / \mathrm{mL}(\mathrm{p}=0.024)$. The discordance in the GS between biopsy and RRP specimens is widely recognized, but the prognostic importance of these discrepancies has not been definitively established. However, Boorijan et al. (27) published one of the few studies showing that discordance in the GS is associated with adverse pathological features, including advanced tumor stage, lymph node metastasis and positive surgical margins. Chun et al. concluded that the tumor weight is an independent prognostic indicator of biochemical recurrence (6). In our study, the significant rate of agreement was found exactly in the subgroup with variables indicative of good prognosis (prostate weight $<50 \mathrm{~g}$ and PSA $<10 \mathrm{ng}$ / $\mathrm{mL}$ ), but this subgroup corresponds to only $37 \%$ of all single positive core patients in this study. This result suggests that single positive core PCa cannot be considered indolent.

AS is an approach for the management of low-risk PCa that can help avoid or delay treatment with RP or radiotherapy. A previous study showed that $96 \%$ of patients with a negative repeat biopsy had only a single positive core in the initial biopsy, whereas 32\% who had upstaging/ upgrading due to the repeat biopsy had 2 or 3 positive cores in the initial biopsy during the AS. However, the treated patients who were upgraded and/or understaged were more likely to have a higher pathological stage and grade at RRP than those who were not upgraded and/or understaged ( $p=0.003$ and $p=0.001$, respectively) (28). Our study, which most likely includes the largest case series ever reported, showed that two-thirds of single positive core cT1 tumors became bilateral or locally advanced tumors (pT3) and that 68\% (106/156) of patients with AS criteria had pT2c-pT3 tumors at RRP. Should we even consider following these patients?

Many studies have focused on single positive core prostate biopsies as a potential variable in the identification of insignificant PCa. The presence of PSMs is considered a poor prognostic factor in patients after RRP, but the impact on clinical end points is a topic of ongoing discussion (29). Mauermann found that $34.5 \%$ of patients with
pT2-T4 had PSMs (29). Taverna reported PSMs in $29.1 \%$ of a population composed of 134 patients with microfocus prostate adenocarcinomas (4). We found PSMs in 20.9\% of our patients, and the rate of PSMs increased with increasing GS. There was a significant difference between Gleason 8 patients and Gleason 6 and 7 patients $(\mathrm{p}=0.013$ and 0.021 , respectively).

A recent study of patients who were eligible for AS but underwent RRP found that 12.5\% had EPE and only $26 \%$ of patients had "insignificant" tumors $(30,31)$, very similar to the results of our study. Here again, the EPE rate increased with increasing GS, with a statistically significant difference between Gleason 8 patients and Gleason 6 and 7 patients $(\mathrm{p}=0.003$ and 0.014 , respectively). We found the same rate of SVI for all GSs, and the observed prevalence was similar to that reported in the literature, which is approximately 5.0\% for very low tumors. EPE, PSMs and SVI are associated with an increased risk of biochemical recurrence and $\mathrm{PCa}$-specific death.

One strength of our study is the large number of patient and the very detailed analysis. The retrospective design and the lack of a standardized biopsy pathological review are the main limitations of the current study.

\section{CONCLUSIONS}

Patients with single positive core PCa, independent of the GS, were found to have clinically significant disease when the RRP specimens were analyzed, with considerable overgrading of GS, pT2c-pT3, PSMs, EPE and SVI. These characteristics suggest that single positive core $\mathrm{PCa}$ should be analyzed individually, should be discussed with the patient and can be considered for treatment in the same manner as multiple positive core $\mathrm{PCa}$, except for the cases with PSA $<10$ and prostate $<$ $50 \mathrm{~g}$, where we find more concordance between biopsy and RRP specimen.

\section{CONFLICT OF INTEREST}

None declared. 


\section{REFERENCES}

1. Siegel R, DeSantis C, Virgo K, Stein K, Mariotto A, Smith T, et al.: Cancer treatment and survivorship statistics, 2012. CA Cancer J Clin. 2012; 62: 220-41.

2. Al Otaibi M, Ross P, Fahmy N, Jeyaganth S, Trottier H, Sircar K, et al.: Role of repeated biopsy of the prostate in predicting disease progression in patients with prostate cancer on activesurveillance. Cancer. 2008; 113: 286-92.

3. Schröder FH, Hugosson J, Roobol MJ, Tammela TL, Ciatto $\mathrm{S}$, Nelen V, et al.: Prostate-cancer mortality at 11 years of follow-up. N Engl J Med. 2012; 366: 981-90. Erratum in: N Engl J Med. 2012; 366: 2137.

4. Taverna G, Benecchi L, Grizzi F, Seveso M, Giusti G, Piccinelli A, et al.: Can a Gleason 6 or Less Microfocus of Prostate Cancer in One Biopsy and Prostate-Specific Antigen Level $<10 \mathrm{ng} / \mathrm{mL}$ Be Defined as the Archetype of Low-Risk Prostate Disease? J Oncol. 2012; 2012: 645146.

5. Ahn HJ, Ko YH, Jang HA, Kang SG, Kang SH, Park HS, et al.: Single positive core prostate cancer in a 12-core transrectal biopsy scheme: clinicopathological implications compared withmultifocal counterpart. Korean J Urol. 2010; 51: 671-6.

6. Chun FK, Briganti A, Gallina A, Hutterer GC, Shariat SF, Antebie E, et al.: Prostate-specific antigen improves the ability of clinical stage and biopsy Gleason sum to predict the pathologic stage at radical prostatectomy in the new milIennium. Eur Urol. 2007; 52: 1067-74.

7. Cooperberg MR, Broering JM, Litwin MS, Lubeck DP, Mehta SS, Henning JM, et al.: The contemporary management of prostate cancer in the United States: lessons from the cancer of the prostate strategic urologic research endeavor (CapSURE), a national disease registry. J Urol. 2004; 171: 1393-401.

8. Makarov DV, Trock BJ, Humphreys EB, Mangold LA, Walsh PC, Epstein JI, et al.: Updated nomogram to predict pathologic stage of prostate cancer given prostate-specific antigen level, clinical stage, andbiopsy Gleason score (Partin tables) based on cases from 2000 to 2005. Urology. 2007; 69: 1095-101.

9. Bill-Axelson A, Holmberg L, Ruutu M, Garmo H, Stark JR, Busch $C$, et al.: Radical prostatectomy versus watchful waiting in early prostate cancer. N Engl J Med. 2011; 364: 1708-17.

10. Wilt TJ, Brawer MK, Jones KM, Barry MJ, Aronson WJ, Fox S, et al.: Radical prostatectomy versus observation for localized prostate cancer. N Engl J Med. 2012; 367: 203-13. Erratum in: N Engl J Med. 2012; 367: 582.

11. Moyer VA; U.S. Preventive Services Task Force: Screening for prostate cancer: U.S. Preventive Services Task Force recommendation statement. Ann Intern Med. 2012; 157: 120-34.
12. Porten SP, Whitson JM, Cowan JE, Perez N, Shinohara K, Carroll PR: Changes in cancer volume in serial biopsies of men on active surveillance for early stage prostate cancer. J Urol. 2011; 186: 1825-9.

13. Dall'Era MA, Konety BR, Cowan JE, Shinohara K, Stauf F, Cooperberg MR, et al.: Active surveillance for the management of prostate cancer in a contemporary cohort. Cancer. 2008; 112: 2664-70.

14. Warlick C, Trock BJ, Landis P, Epstein JI, Carter HB: Delayed versus immediate surgical intervention and prostate cancer outcome. J Natl Cancer Inst. 2006; 98: 355-7.

15. Truesdale MD, Cheetham PJ, Turk AT, Sartori S, Hruby GW, Dinneen EP, et al.: Gleason score concordance on biopsyconfirmed prostate cancer: is pathological re-evaluation necessary prior to radicalprostatectomy? BJU Int. 2011; 107: 749-54.

16. Epstein JI, Allsbrook WC Jr, Amin MB, Egevad LL; ISUP Grading Committee: The 2005 International Society of Urological Pathology (ISUP) Consensus Conference on Gleason Grading of ProstaticCarcinoma. Am J Surg Pathol. 2005; 29: 1228-42.

17. Epstein JI, Walsh PC, Carmichael M, Brendler CB: Pathologic and clinical findings to predict tumor extent of nonpalpable (stage T1c) prostate cancer. JAMA. 1994; 271: 368-74.

18. Kerkhof M, Roobol MJ, Cuzick J, Sasieni P, Roemeling S, Schröder FH, et al.: Effect of the correction for noncompliance and contamination on the estimated reduction of metastatic prostate cancer within a randomized screening trial (ERSPC section Rotterdam). Int J Cancer. 2010; 127: 2639-44.

19. Brawer MK, The Influence of Prostate Volume on Prostate Cancer Detection. Eur Urol Supplements. 2002; 1: 35-9.

20. Karakiewicz PI, Bazinet M, Aprikian AG, Trudel C, Aronson $S$, Nachabé M, et al.: Outcome of sextant biopsy according to gland volume. Urology. 1997; 49: 55-9.

21. Divrik RT, Eroglu A, Sahin A, Zorlu F, Ozen H: Increasing the number of biopsies increases the concordance of Gleason scores of needle biopsies and prostatectomy specimens. Urol Oncol. 2007; 25: 376-82.

22. Sved PD, Gomez P, Manoharan M, Kim SS, Soloway MS: Limitations of biopsy Gleason grade: implications for counseling patients with biopsy Gleason score 6 prostate cancer. J Urol. 2004; 172: 98-102.

23. Karavitakis M, Ahmed HU, Abel PD, Hazell S, Winkler MH: Anatomically versus biologically unifocal prostate cancer: a pathological evaluation in the context of focal therapy. Ther Adv Urol. 2012; 4: 155-60.

24. Ishizaki F, Hara N, Koike H, Kawaguchi M, Tadokoro A, Takizawa I, et al.: Prediction of pathological and oncological outcomes based on extended prostate biopsy results in patients with prostate cancer receiving radical prostatectomy: a single institution study. Diagn Pathol. 2012; 7: 68. 
25. King CR, McNeal JE, Gill H, Presti JC Jr.: Extended prostate biopsy scheme improves reliability of Gleason grading: implications for radiotherapy patients. Int J Radiat Oncol Biol Phys. 2004; 59: 386-91.

26. Boorjian SA, Karnes RJ, Crispen PL, Rangel LJ, Bergstralh EJ, Blute ML: Radiation therapy after radical prostatectomy: impact on metastasis and survival. J Urol. 2009; 182: 2708-14.

27. Berglund RK, Masterson TA, Vora KC, Eggener SE, Eastham JA, Guillonneau BD: Pathological upgrading and up staging with immediate repeat biopsy in patients eligible for active surveillance. J Urol. 2008; 180: 1964-7; discussion 1967-8.

28. Mauermann J, Fradet V, Lacombe L, Dujardin T, Tiguert $\mathrm{R}$, Tetu $\mathrm{B}$, et al.: The impact of solitary and multiple positive surgical margins on hard clinical end points in 1712 adjuvant treatment-naive pT2-4 NO radical prostatectomy patients. Eur Urol. 2013; 64: 19-25.
29. Beauval JB, Ploussard G, Soulié M, Pfister C, Van Agt S, Vincendeau $S$, et al.: Pathologic findings in radical prostatectomy specimens from patients eligible for active surveillance with highly selective criteria: a multicenter study. Urology. 2012; 80: 656-60.

30. Katz B, Srougi M, Camara-Lopes LH, Antunes AA, Nesrallah $L$, Nesrallah $A$, et al.: The accuracy of pathological data for the prediction of insignificant prostate cancer. Int Braz $\mathrm{J}$ Urol. 2012; 38: 760-8.

Correspondence address:

Ricardo Kupka da Silva, MD

Division of Uro-Oncology

University of São Paulo Medical School Av. Dr Arnaldo, $251 / 4^{\circ}$ andar, Cerqueira César

São Paulo, SP, 01246-000, Brazil E-mail: ricardokupka@hotmail.com Fax: + 5511 3893-2528 